\title{
Estimation des niveaux d'inondation pour une crue éclair en milieu urbain : comparaison de deux modèles hydrodynamiques sur la crue de Nîmes d'octobre 1988 Estimation of the flood levels for a flash flood in urban area : comparison of two hydrodynamic models on the 1988's Nîmes flood
}

\author{
A. Paquier, J. M. Tanguy, S. Haider et B. Zhang
}

Volume 16, numéro 1, 2003

URI : https://id.erudit.org/iderudit/705499ar

DOI : https://doi.org/10.7202/705499ar

Aller au sommaire du numéro

\section{Éditeur(s)}

Université du Québec - INRS-Eau, Terre et Environnement (INRS-ETE)

ISSN

0992-7158 (imprimé)

1718-8598 (numérique)

Découvrir la revue

Citer cet article

Paquier, A., Tanguy, J. M., Haider, S. \& Zhang, B. (2003). Estimation des niveaux d'inondation pour une crue éclair en milieu urbain : comparaison de deux modèles hydrodynamiques sur la crue de Nîmes d'octobre 1988. Revue des sciences de l'eau / Journal of Water Science, 16(1), 79-102. https://doi.org/10.7202/705499ar

\section{Résumé de l'article}

Lors des crues extrêmes en ville, une forte part des écoulements reste en surface. Pour simuler ces inondations, deux modèles sont présentés : le logiciel $\mathrm{REM}^{2} \mathrm{U}$ unidimensionnel a pour objectif de simuler la propagation des débits de crue dans l'ensemble d'un réseau de rues alors que le logiciel Rubar 20 bidimensionnel vise à fournir plus d'information sur ces écoulements. Des calculs avec ces deux logiciels ont été menés sur la crue d'octobre 1988 dans un quartier de Nîmes. Lors de cet événement, les hauteurs d'eau maximales ont dépassé deux mètres en certains points et les vitesses $2 \mathrm{~m} / \mathrm{s}$ ce qui entraînait des passages en régime torrentiel. A partir des données rassemblées sur les sections en travers des rues, des maillages de calcul limités au réseau de rues ont été construits pour les deux logiciels afin de permettre un calcul détaillé. La comparaison des résultats avec les laisses de crue montre des situations très contrastées d'un point à un autre pour une hauteur d'eau maximale moyenne sur l'ensemble de la zone inondée correctement simulée. L'écart sur cette hauteur est, en moyenne, de $1 \mathrm{~m}$ ce qui provient des incertitudes sur les observations, sur la topographie et sur les conditions aux limites, des approximations lors de la modélisation et de particularités locales non décrites. Entre les deux logiciels, l'évolution des hauteurs et des vitesses est généralement très proche bien que, comme pour la comparaison avec les laisses de crue, des différences locales importantes sont observées. 


\section{Estimation des niveaux d'inondation pour une crue éclair en milieu urbain : comparaison de deux modèles hydrodynamiques sur la crue de Nîmes d'octobre 1988}

\section{Estimation of the flood levels for a flash flood in urban area: comparison of two hydrodynamic models on the 1988's Nîmes flood}

\section{A. PAQUIER ${ }^{1}{ }^{*}$, J.M. TANGUY ${ }^{2}$, S. HAIDER ${ }^{1}$, B. ZHANG ${ }^{3}$}

Reçu le 14 mars 2002, accepté le 19 novembre $2002^{\star \star}$.

\section{SUMMARY}

The hydraulic models that are used to simulate floods in rural areas are not adapted to model floods through urban areas, because of details that may deviate flows and create strong discontinuities in the water levels, and because of the possible water flow running in the sewage network. However, such modelling is strongly required because damage is often concentrated in urban areas. Thus, it is necessary to develop models specifically dedicated to such floods. In the southern part of France, rains may have a high intensity but floods generally last a few hours. During extreme events such as the October 1988 flood in the city of Nîmes, most of the flow remained on the ground with high water depths and high velocities, and the role of sewage network can be neglected. A 1-D model and a 2-D model were used to calculate such flows, which may become supercritical. On the catchments of the streams which cross the city of Nîmes, the rainfall was estimated as $80 \mathrm{~mm}$ in one hour and $250 \mathrm{~mm}$ in six hours in October 1988, although some uncertainties remain. The return period can be estimated between 150 and 250 years. The zone selected to test the models was an area $1.2 \mathrm{~km}$ long and less than $1 \mathrm{~km}$ wide in the north-eastern part of the city. It includes a southern part with a high density of houses. The slope from the North (upstream) to the South (downstream) was more than $1 \%$ on average and was decreasing from North to South. Various topographical and hydrological data were obtained from the local Authorities. The basic data were composed of 258 cross sections of 69 streets with 11 to 19 points for each cross section. Observations of the limits of the flooded areas and of the peak water levels at more than 80 points can be used to validate the calculation results. The inputs consisted of two discharge hydrographs, estimated from a rainfall-discharge model from rains with a return period of 100 years, which may result in an unde-

1. Cemagref, Unité de recherche hydrologie-hydraulique, CP 22069336 Lyon cedex 09.

2. CETE Méditerranée : BP 37000, 13791 Aix-en-Provence cedex 3.

3. GRADIENT Groupe université de technologie de Compiègne, 66, rue de Lanshut, 60200 Compiègne.

* Correspondance. E-mail : andre.paquier@cemagref.fr

** Les commentaires seront reçus jusqu'au 30 septembre 2003. 
restimate of these inputs. These two hydrographs correspond to the two main structures that cross the railway embankment, which constitutes an impervious upstream boundary of the modelled area. Whereas the western and eastern boundaries are well delimitated by hills above maximum water levels, the downstream southern boundary is somewhat more questionable because of possibilities of backwater and inflows from neighbouring areas.

The 1-D software $R E M^{2} U$ solved the Saint Venant equations on a meshed network. At crossroads, continuities of discharge and of water heads were set. The hydraulic jump was modelled by a numerical diffusion applied wherever high water levels were found. The Lax Wendroff numerical scheme was implemented. It included a prediction step and a correction step, which implied precise solving of these very unsteady and hyperbolic problems. The software was validated on numerous test cases (AL MIKDAD, 2000) which proved the adaptation to problems of calculations in a network of streets.

The 2-D software Rubar 20 solves 2-D shallow water equations by an explicit second-order Van Leer type finite volume scheme on a computational grid made from triangles and quadrilaterals (PAQUIER, 1998). The discontinuities (hydraulic jumps for instance) are treated as ordinary points through the solving of Riemann problems. For the Nîmes case, the grid was built from the cross sections of the streets. Four grids were built with respectively $4,5,7$ or 11 points for every cross section and these points correspond to the main characteristics of the cross section: the walls of the buildings, the sidewalks, the gutters and the middle point. The simplest crossroads were described from the crossings of the lines corresponding to these points, which provide respectively $9,16,36$ or 100 computational cells. The space step was about 25 metres along the streets but went as low as $\mathbf{0 . 1} \mathrm{m}$ in the crossroads; due to the explicit scheme, which implies that the Courant number was limited to 1 , the time step was very small and a long computational time was required.

The computations were performed with a uniform Strickler coefficient of $40 \mathrm{~m}^{1 / 3} / \mathrm{s}$. Both 1-D and 2-D models provided results that agreed well with observed water levels. The limits of the flooded area were also quite well simulated. However, locally, the differences between calculated and observed maximum water depths were high, resulting in an average deviation of about 1 metre. The reasons for such deviations could come from three main causes. First, the uncertainty of topographical data is relatively high, because of the interpolation between measured cross sections without a detailed complementary DEM (digital elevation model). Second, the observed levels were also uncertain and reveal local situations that are not reconstructed by the hydraulic models which provided maxìmum water levels averaged on one cell which may not coincide with the exact location of the observations. Finally, modelling means a simplification of the processes, which implies cancelling the level variations due to some obstacles, such as cars, which are not simple to identify.

In conclusion, both software packages can model a flood, even a flash flood, in an urbanised area. Research is still necessary to develop methods to fully use urban databases in order to define details more precisely. The improvements to the 1-D software should include a better modelling of storage and of crossroads with an integration of adapted relations for the head losses. 2-D software has a greater potential but the difficulty to build an optimal computational grid means a long computational time, which limits the use of such software to small areas. For both software packages, methods still need to be developed in order to represent exchanges with the sewage network, storage inside buildings and inputs directly coming from rainfall.

Key-words: flash floods, urban risk, hydrodynamic modelling, de Saint Venant equations, October 1988 Nîmes flood, l-D software, 2-D software, hydraulic jump. 


\section{RÉSUMÉ}

Lors des crues extrêmes en ville, une forte part des écoulements reste en surface. Pour simuler ces inondations, deux modèles sont présentés : le logiciel $\mathbf{R E M}^{2} \mathrm{U}$ unidimensionnel a pour objectif de simuler la propagation des débits de crue dans l'ensemble d'un réseau de rues alors que le logiciel Rubar 20 bidimensionnel vise à fournir plus d'information sur ces écoulements. Des calculs avec ces deux logiciels ont été menés sur la crue d'octobre 1988 dans un quartier de Nîmes. Lors de cet événement, les hauteurs d'eau maximales ont dépassé deux mètres en certains points et les vitesses $2 \mathrm{~m} / \mathrm{s}$ ce qui entrấnait des passages en régime torrentiel. À partir des données rassemblées sur les sections en travers des rues, des maillages de calcul limités au réseau de rues ont été construits pour les deux logiciels afin de permettre un calcul détaillé. La comparaison des résultats avec les laisses de crue montre des situations très contrastées d'un point à un autre pour une hauteur d'eau maximale moyenne sur l'ensemble de la zone inondée correctement simulée. L'écart sur cette hauteur est, en moyenne, de $1 \mathrm{~m}$ ce qui provient des incertitudes sur les observations, sur la topographie et sur les conditions aux limites, des approximations lors de la modélisation et de particularités locales non décrites. Entre les deux logiciels, l'évolution des hauteurs et des vitesses est généralement très proche bien que, comme pour la comparaison avec les laisses de crue, des différences locales importantes sont observées.

Mots clés : crues éclair, risques urbains, modélisation hydraulique, équations de Saint Venant, crue de Nîmes d'octobre 1988, logiciel ID, logiciel 2D, ressaut hydraulique.

\section{1 - INTRODUCTION}

En France, les inondations sont la cause principale des dommages consécutifs à des événements d'origine naturelle. L'augmentation du montant des dommages est, en grande partie, liée à l'urbanisation dans des zones inondables. Afin d'éviter les pertes en vies humaines, la mise en place de systèmes d'annonce de crue a été accélérée. Leur efficacité ainsi que le développement de mesures de prévention seules susceptibles de réduire fortement les dommages économiques directs et indirects repose sur une estimation la plus détaillée possible des risques d'inondation. Les modélisations numériques utilisées en zone rurale pour des crues souvent lentes (et généralement fondées sur un réseau ramifié de biefs unidimensionnels en régime fluvial) sont relativement inefficaces pour simuler les processus hydrauliques qui se produisent en zone urbaine : les multiples obstacles dérivent brutalement les écoulements, ce qui peut conduire à la formation de vagues et à des passages en régime torrentiel. Ces phénomènes sont accentués en milieu méditerranéen où les pluies sont plus violentes et les pentes plus fortes. Des méthodes de calcul spécifiques doivent donc être développées. Elles sont, en particulier, nécessaires pour modéliser le régime torrentiel et le passage du régime torrentiel au régime fluvial (et inversement) qui est à l'origine de ressauts hydrauliques qui se produisent parfois près des carrefours du fait des élargissements et rétrécissements relativement brusques.

Dans ces cas, les approximations effectuées par différents auteurs pour la modélisation des écoulements en ville (par exemple, le régime uniforme pour 
SMITH, 1992 ; la suppression du terme convectif pour GALLATI et BRASCHI, 1990 ; la représentation par des cellules connectées par des débits d'échange pour RICCARDI, 1997) semblent inacceptables pour obtenir des résultats significatifs localement et le recours aux équations de Saint Venant complètes qui n'effectuent pas ces simplifications est nécessaire. II demeure que les situations sont très diversifiées en zone urbaine et que, dans un objectif de modélisation sur l'ensemble d'une ville, il est surtout important de bien représenter l'organisation générale des bâtiments (HERVOUET et al., 2000 ; HINGRAY et al., 2000 ; LAl et al., 2000) voire celle du bassin versant (CAMPANA et al., 2001).

Nous présentons ci-après deux méthodes de calcul, l'une unidimensionnelle, l'autre bidimensionnelle qui permettent ce type de modélisation ainsi que leur application à un cas d'inondation extrême, la crue du 3 octobre 1988 dans la ville de Nîmes. Cette application a été effectuée dans le cadre du projet "Risques hydrologiques en milieu urbain » du programme Risques d'inondation du ministère de l'Aménagement du Territoire et de l'Environnement français qui avait pour objectif de déterminer les possibilités des simulations numériques des inondations dans ce contexte urbain. La modélisation bidimensionnelle devait a priori servir de référence par sa capacité potentielle à décrire explicitement les écoulements en présence de bâtiments et singularités diverses ; dans l'exemple décrit où la modélisation est limitée au réseau de rues, c'est surtout la capacité à traiter les carrefours qui est utilisée. Pour ce dernier aspect, une validation du calcul bidimensionnel pour des jonctions ou dérivations en canal rectangulaire existe à travers plusieurs expériences (par exemple KHAN et al., 2000). La modélisation unidimensionnelle, en revanche, devait préfigurer un outil utilisable sur un ensemble de rues plus vaste.

Après une description du contexte de l'exemple traité, des données correspondantes et des deux modèles utilisés REM2U unidimensionnel et RUBAR 20 bidimensionnel, l'analyse des résultats des calculs met en lumière les possibilités de modélisation (avec un niveau de détail variable pour RUBAR 20) et les limitations atteintes lors de la simulation d'une crue éclair en milieu urbain.

\section{2 - INONDATION DU 3 OCTOBRE 1988 À NÎMES}

\subsection{Pluies}

À travers l'histoire, la ville de Nîmes a subi de nombreuses inondations. Un inventaire de différentes sources historiques (DESBORDES et al., 1989) a dénombré 39 crues conséquentes entre 1334 et 1988 soit une périodicité d'une crue tous les 17 ans. Les $X V I^{e}$ et $X I X^{e}$ siècles totalisent plus de $50 \%$ de ces crues.

L'inondation d'octobre 1988 est consécutive à un événement pluvieux d'une ampleur exceptionnelle qui a fait déborder les " cadereaux », ruisseaux nîmois encaissés dans les vallons en amont de la ville puis traversant les zones urbanisées souvent par des conduites.

Bien que les enregistrements varient d'un pluviomètre à l'autre, de plus fortes intensités ayant sans doute frappé le Nord de la ville (Mas de Ponge), il peut être 
retenu, en moyenne, sur l'ensemble des bassins versants, des hauteurs de l'ordre de $80 \mathrm{~mm}$ en une heure, $150 \mathrm{~mm}$ en trois heures et $250 \mathrm{~mm}$ en six heures pour la période des précipitations intenses. Une analyse statistique des pluies sur une durée de l'ordre de grandeur de la durée caractéristique des bassins versants (soit 2 à 6 heures) donne une période de retour de l'ordre de 150 à 250 ans mais peut être abaissée fortement en prenant en compte l'information régionale et l'information historique (DESBORDES et al., 1989). Comme le laissait entrevoir l'analyse historique, il s'agit d'un événement relativement fréquent pour lequel des mesures de prévention contre les inondations sont nécessaires.

\subsection{Description de la zone modélisée}

Pour un premier test des méthodes de calcul, il avait été décidé de se limiter à une zone restreinte mais pour laquelle le plus grand nombre de données était disponible. Le quartier Richelieu sélectionné se situe au Nord-Est du centre ville (figure 1). Si on se réfère aux crues historiques, il apparaît que des hauteurs d'eau de l'ordre d'un mètre ou plus ont été relevées à au moins cinq reprises ces 150 dernières années. Pour la crue d'octobre 1988, les hauteurs d'eau ont dépassé deux mètres en plusieurs points mais les hauteurs d'eau maximales dans les rues sont de l'ordre d'un mètre en moyenne.

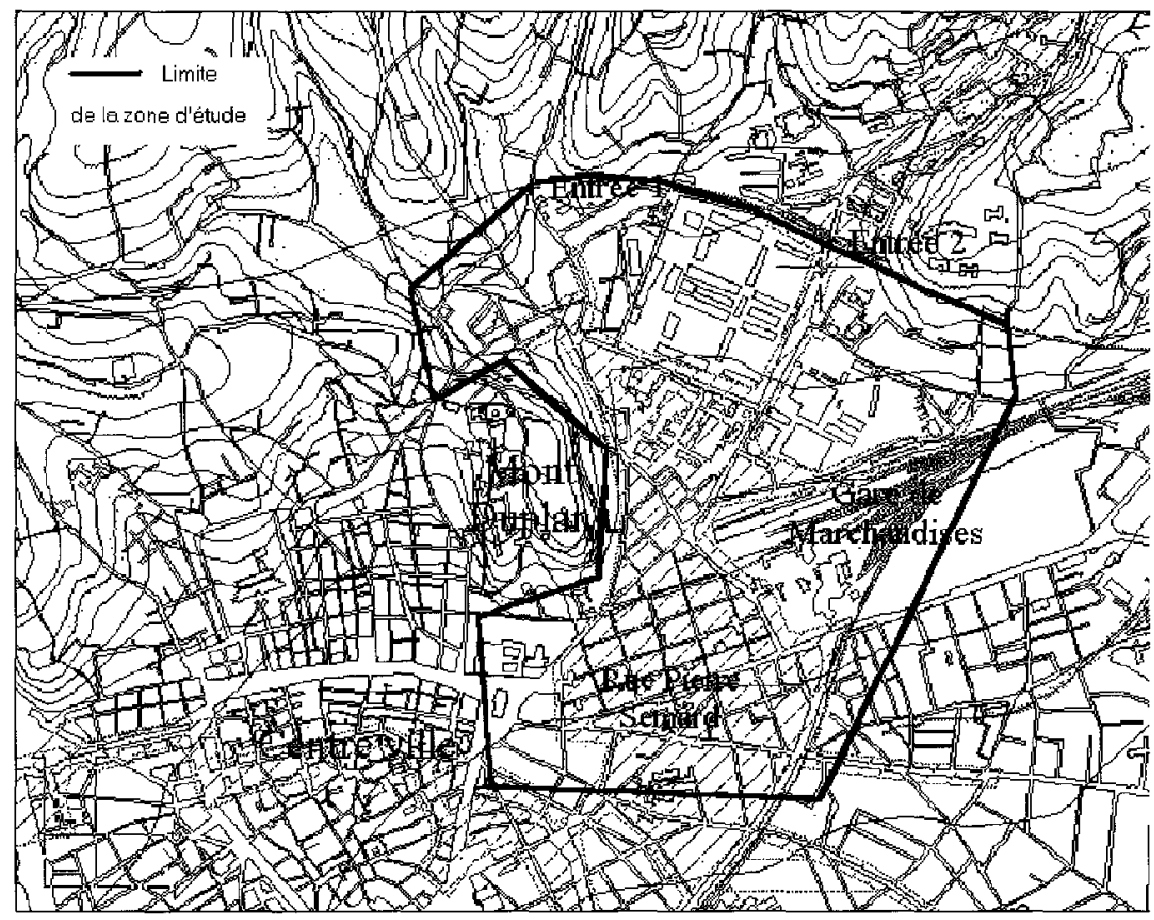

Figure 1 Plan de situation (extrait du plan fourni par la DDE du Gard).

Situation map (extracted from the map provided by the Gard DDE). 
La zone modélisée est limitée au Nord par une voie ferrée en remblai à la traversée des talwegs ce qui permet, en première approximation, de réduire les apports aux deux points correspondant respectivement au chemin des Limites (noté « entrée 1 » sur la figure 1) et au cadereau d'Uzès (« entrée 2 »). À l'Ouest, le Mont Duplan et, à l'Est, la gare de marchandises bâtie sur un remblai constituent des limites « naturelles » des inondations. C'est uniquement au Sud vers la rue Pierre Semard que les échanges avec les zones avoisinantes également inondées sont mal connus.

La totalité de la zone considérée a une longueur de 1200 mètres environ et une largeur très variable avec un maximum de l'ordre de 1000 mètres. La direction de l'écoulement est grossièrement Nord-Sud depuis l'entrée aux deux passages sous la voie ferrée jusqu'à l'étalement au Sud de la rue Pierre Semard.

Le dénivelé entre l'amont et l'aval de la zone varie en fonction du point d'entrée considéré. À l'Ouest, le dénivelé est de 18 mètres environ alors qu'il n'est que de 13 mètres à l'Est. Dans les deux cas, la pente moyenne est supérieure à $1 \%$ et s'atténue en allant du Nord vers le Sud. Par ailleurs, le quartier choisi peut se décomposer en une partie Nord qui comprend des zones de faible occupation du sol (grands immeubles d'habitation, casernes, hôpital...) et la partie Sud qui est une zone d'habitat dense avec un ensemble de rues relativement étroites (jusqu'à moins de 6 mètres de large) se croisant à angle droit.

\subsection{Données de base des modélisations}

La modélisation s'est appuyée sur un ensemble de données fournies par la DDE (Direction départementale de l'équipement) du Gard et la ville de Nîmes et exploitées par le CETE (Centre technique de l'équipement) de Bordeaux. Nous récapitulons ci-dessous les principales données.

La description du réseau de rues a été effectuée à travers un ensemble de profils en travers de rues. Cet ensemble comprend un total de 258 profils pour 69 rues. Le nombre de profils par rue varie de 2 a 10. La plupart des rues sont décrites par deux profils mais les rues principales avec des changements de direction et de largeur exigent plus de profils. Chaque profil en travers est constitué de 11 à 19 points définis par leurs trois coordonnées Lambert $x, y$ et $z$. Les points fournis correspondent à des détails topographiques : bâtiment en bordure de rue, trottoir, caniveau, chaussée. La description topographique a été complétée par des points cotés éparpillés sur l'ensemble de la zone, ces derniers ont pu être exploités en complément lorsque l'interpolation entre profils en travers était insuffisante ou erronée.

La carte des zones inondées lors de la crue d'octobre 1988 comporte le contour approximatif de l'inondation (voir figure 6) ainsi que l'emplacement d'environ 80 points d'observation. En chacun de ces derniers points, avaient été relevées, juste après la crue, les laisses de crue (correspondant plus ou moins au niveau maximum) et les cotes du sol aux mêmes points. Ces points ainsi que la limite d'inondation permettent une validation des résultats des calculs.

Les hydrogrammes (aux entrées 1 et 2) fournis par la DDE n'étaient pas issus de mesures de débit effectuées en un point donné mais provenaient d'une transformation pluie-débit sur les bassins versants jusqu'à leur point de 
jonction naturel au droit de la gare de marchandises (au centre de la zone modélisée). Ces hydrogrammes concernent une pluie de période de retour de 100 ans et nous avons estimé que, lors de la crue d'octobre 1988, I'hydrogramme réel était compris entre l'hydrogramme simulé et un hydrogramme de l'ordre de $40 \%$ supérieur. Ceci doit permettre d'inclure l'incertitude sur la pluie et sur l'extension du bassin versant, cette dernière étant accrue par le fait que les apports intermédiaires en provenance de la zone modélisée elle-même et des abords n'ont pas été pris en compte. Les deux hydrogrammes ont été fournis sur une durée de sept heures avec un pas de temps de 5 minutes (figure 2). L'hydrogramme 1 a un pic de $43,0 \mathrm{~m}^{3} / \mathrm{s}$ et l'hydrogramme 2 un pic de $49,8 \mathrm{~m}^{3} / \mathrm{s}$. Les pointes de débit supérieures d'au moins un ordre de grandeur aux débits transités par le réseau d'assainissement justifient de négliger ce réseau dans la modélisation. Afin de réduire le temps de calcul, la durée de simulation a été limitée à quatre heures de crue. Ce temps correspond à la phase de décrue et une simulation jusqu'à ce temps suffit pour calculer l'étendue maximale de la crue ainsi que les niveaux maximaux en tout point.

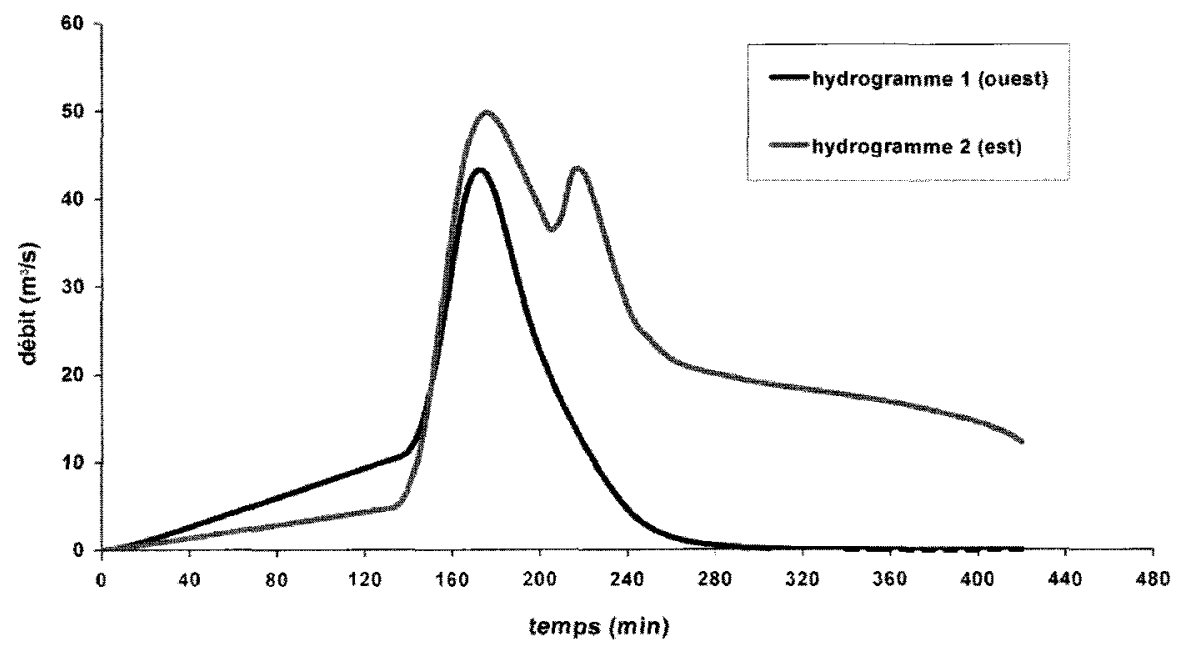

Figure 2 Les hydrogrammes utilisés pour les simulations.

The discharge hydrographs used for the calculations.

\section{3 - MODÉLISATION UNIDIMENSIONNELLE}

\subsection{Présentation du logiciel}

Le modèle unidimensionnel REM2U résout les équations unidimensionnelles de l'hydraulique à surface libre (équations de Saint Venant) en réseau maillé, que nous décrivons ici sous leur forme conservative (inconnues : débit $Q$ et niveau d'eau $h$ ). 


\subsection{1 Équation de continuité}

$$
\frac{\partial(B h)}{\partial t}+\frac{\partial Q}{\partial x}=u_{p} B
$$

Le terme de droite de cette équation permet de prendre en compte les apports de pluie directement dans les rues, mais également en provenance des toitures des habitations. Ces eaux rejoignent les ncuds du maillage. Cependant, dans le cas de Nîmes, cette option n'a pas été utilisée.

\subsection{2 Équation de conservation de la quantité de mouvement}

$$
\frac{\partial Q}{\partial t}+\frac{\partial\left(Q^{2} / A\right)}{\partial x}+g \frac{\partial l}{\partial x}+g A\left(S_{f}-S_{0}\right)-\frac{\partial}{\partial x}\left(v_{t} A \frac{\partial}{\partial x}\left(\frac{Q}{A}\right)\right)=-u_{p} \frac{Q}{H}
$$

où $B$ est la largeur au miroir de la section du canal, $h$ la cote de la surface libre, $A$ la section mouillée de la rue, $Q$ le débit, $S_{0}$ la pente du fond, $u_{p}$ l'intensité des précipitations, $S_{f}$ la pente de frottement, $H$ la profondeur d'eau, $v_{t}$ la viscosité que nous préférons nommer « dispersion ", I la pression hydrostatique et $g$ l'accélération de la pesanteur. Le terme de droite de cette équation résulte de l'intégration dans la verticale des équations de Saint Venant.

Dans l'état actuel de développement du modèle, les profils sont rectangulaires, définis latéralement par les façades des maisons et la chaussée comme plancher.

\subsubsection{Continuité de débit à chaque jonction (ou carrefour)}

Au niveau de chaque jonction, la continuité des débits est assurée sur l'ensemble des branches reliées à cette jonction :

$$
\sum_{j=1}^{j=n b b r a n c h e s} Q_{j} n_{j}=0
$$

où $Q_{j}$ est le débit de la branche $j$ arrivant à la jonction $i$ et $n_{j}$ la normale sortante de la branche $j$.

\subsection{4 Équation de continuité de la charge dans un carrefour}

L'expression de la perte de charge entre les branches $j$ et $k$ est donnée par l'expression suivante :

$$
h_{j}-h_{k}+\beta Q_{k}^{2}=0
$$

où $h_{j}$ est la charge dans la branche $j$ et $\beta$ un paramètre dépendant du coefficient de perte de charge. Dans l'état actuel de développement du modèle, $\beta=0$. Des essais sur modèle physique vont être réalisés pour quantifier ce coefficient dans le cas de secteurs urbains aux caractéristiques différentes (tissus urbains anciens ou quartiers neufs). 


\subsubsection{Capture du ressaut hydraulique}

L'hétérogénéité des caractéristiques topologiques et topographiques des réseaux de voiries en milieu urbain met en œuvre la plupart des types de singularités hydrauliques : rétrécissements progressifs ou contractions brutales, élargissements, seuils, pentes faibles ou fortes, etc. Ceci induit des régimes hydrauliques successivement fluviaux et torrentiels et exige donc une modélisation des transitions de l'un à l'autre. Si la transition fluvial-torrentiel ne pose en général pas de problème particulier au niveau numérique, il n'en est pas de même pour le ressaut hydraulique qui s'accompagne d'une forte perte de charge.

Pour être en mesure de reproduire cette perte de charge, le modèle utilise la technique de "capture de choc " (ici capture du ressaut) bien connue dans le domaine des écoulements compressibles qui consiste à introduire une diffusion numérique dans les zones à forts gradients de niveaux.

Ce terme de diffusion est introduit comme correcteur du vecteur solution $U^{t+\Delta t}=\left\{\begin{array}{l}h \\ Q\end{array}\right\}^{t+\Delta t}$ qui résulte de la résolution du système (1) et (2). Le vecteur final corrigé obtenu est le suivant :

$$
U_{c}^{t+\Delta t}=U^{t+\Delta t}+\Delta t \frac{\partial}{\partial x}\left(s_{1_{v}} \frac{\partial U^{t+\Delta t}}{\partial x}\right)
$$

Le coefficient $s_{1 v}$ est donné par le modèle de Lapidus : $s_{i v}=v \Delta x^{2}\left|\frac{\partial u}{\partial x}\right|$ avec $u=\frac{Q}{H}$. $v$ est un terme de diffusion numérique artificielle pris égal à $1 \mathrm{~m}^{2} / \mathrm{s}$ (AL MIKDAD, 2000), $u$ la vitesse, $\Delta x$ la longueur d'un élément, $Q$ le débit et $H$ la profondeur.

\subsubsection{Schéma numérique de résolution}

Le problème à traiter étant de nature transitoire et à forte dominance hyperbolique, le schéma numérique qui est utilisé dans ce modèle est le schéma de Lax-Wendroff, que nous allons décrire synthétiquement ci-après (voir aussi TANGUY et al., 2001 ; AL MIKDAD, 2000).

Le système d'équations (1) et (2) peut s'écrire synthétiquement :

$$
\frac{\partial U}{\partial}+\frac{\partial F}{\partial x}+S+D=P
$$

où $U$ est le vecteur solution, $F$ le vecteur des termes de flux, $S$ le vecteur des termes source, $D$ le vecteur des termes de diffusion.

Avec :

$$
U=\left(\begin{array}{c}
B h \\
Q
\end{array}\right) ; \quad F=\left(\begin{array}{c}
Q \\
\frac{Q^{2}}{A}+g \frac{B H^{2}}{2}
\end{array}\right) ; \quad S=\left(g\left(\frac{\left|\frac{Q}{A}\right| Q}{K^{2} R_{h}^{4 / 3}}-A S_{0}\right)\right)
$$




$$
D=\left(\begin{array}{c}
0 \\
-\frac{\partial}{\partial x}\left(A v_{t} \frac{\partial(Q / A)}{\partial x}\right)
\end{array}\right) ; \quad P=\left(\begin{array}{c}
u_{p} B \\
-u_{p} \frac{Q}{H}
\end{array}\right)
$$

où $K$ est le coefficient de Strickler, $R_{h}$ le rayon hydraulique au droit d'un profil.

La forme variationnelle du système (6) est donnée par :

$$
W=\int_{L}\left(U^{t+\Delta t}-U^{t}\right) d x+\Delta t \int_{L}\left(\frac{\partial F^{t+\frac{\Delta t}{2}}}{\partial x}+S^{t+\frac{\Delta t}{2}}-P^{t+\frac{\Delta t}{2}}+D^{t}\right) d x=0
$$

où $L$ est la longueur du domaine étudié. Rappelons que les grandeurs caractéristiques de l'instant $t+\Delta t / 2$ sont moyennées au niveau de chaque élément.

Le schéma de Lax-Wendroff comporte une étape de prédiction suivie d'une étape de correction qui intègre la capture du ressaut. Ces deux étapes sont incluses dans une même boucle des éléments suivants :

Schéma de Lax-Wendroff

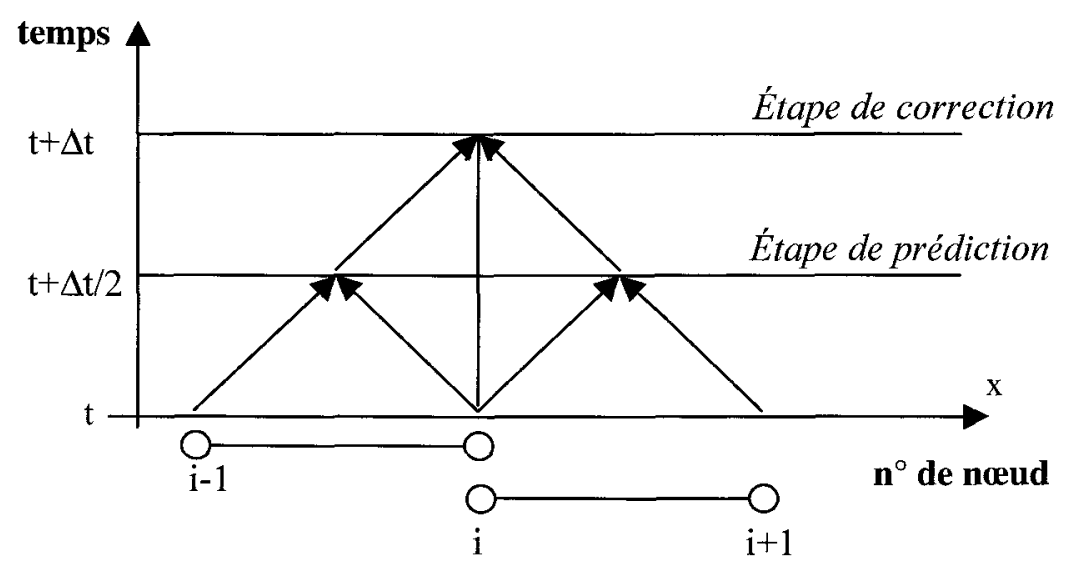

\section{- Étape de Prédiction}

Les valeurs $U^{t+\frac{\Delta t}{2}}, F^{t+\frac{\Delta t}{2}}, S^{t+\frac{\Delta t}{2}}$ et $P^{t+\frac{\Delta t}{2}}$ sont des expressions moyennées, car elles sont calculées au niveau d'un élément à l'instant $t+\Delta t / 2$ comme suit, à partir des expressions (7) pour obtenir finalement :

$$
U^{t+\frac{\Delta t}{2}}=U^{t}-\frac{\Delta t}{2}\left(\frac{\partial F^{t}}{\partial x}+S^{t}-P^{t}\right)
$$

Cette étape de prédiction permet d'économiser beaucoup de temps calcul. Les grandeurs de cette étape peuvent en effet être calculées dans une seule boucle qui intègre les deux étapes. Les termes de diffusion ne sont pas pris en compte à ce niveau. 


\section{- Étape de correction}

La forme variationnelle faible de l'expression (8) est utilisée sur le domaine de longueur $L$ :

$$
\begin{gathered}
W=\int_{L} \delta U\left(U^{t+\Delta t}-U^{t}\right) d x-\Delta t \int_{L} \frac{\partial \delta U}{\partial x}\left(F^{t+\frac{\Delta t}{2}}+\overline{D^{t}}\right) d x \\
+\Delta t \int_{L} \delta U\left(S^{t+\frac{\Delta t}{2}}-P^{t+\frac{\Delta t}{2}}\right) d x+\left[\delta U \Delta t\left(\overline{D^{t}}+F^{t+\frac{\Delta t}{2}}\right)\right]_{x=0}^{x=L}=0
\end{gathered}
$$

avec $\overline{D^{t}}=A^{t} v_{t} \frac{\partial(Q / A)^{t}}{\partial x}$ et $\delta U$ la fonction de pondération de Galerkine.

\section{- Algorithme du schéma de Lax-Wendroff}

L'algorithme suivant est mis en œuvre dans le programme REM ${ }^{2} U$. Les avantages que procure ce schéma (AL MIKDAD, 2000) sont de deux ordres :

- en premier lieu, une faible place en mémoire puisque le schéma totalement explicite ne nécessite d'assembler que la seule matrice masse (devant le terme temporel), les autres entités étant des vecteurs ;

- par ailleurs, les deux premières étapes et notamment l'étape de prédiction qui ne concerne que des grandeurs moyennées sur l'élément peuvent être assemblées dans une même boucle sur les éléments.

De nombreux cas tests assez sévères ont été mis en œuvre dans AL MIKDAD (2000). Ils démontrent une grande précision du schéma ainsi que la possibilité de localiser les ressauts hydrauliques.

\section{Boucle sur les pas de temps}

Assemblage de la matrice masse $M$

Calcul des matrices masses élémentaires

Assemblage des matrices élémentaires dans $M$

Boucle sur les éléments : assemblage du Vecteur Résidu $\mathrm{R}$

Étape de prédiction

Calcul de $U^{t+\frac{\Delta t}{2}}$ par (9)

Étape de correction

Calcul des vecteurs résidus élémentaires par (10) et assemblage dans $R$

Résolution du système $\Delta U=\Delta t M^{-1} R$

Étape de capture de ressaut par (5)

Fin de boucle sur les éléments

\section{Fin de boucle sur les pas de temps}

Le maillage est constitué d'éléments finis linéaires de différentes longueurs délimités par des nœuds dont la densité va dépendre non seulement des caractéristiques topologiques des réseaux de rues, mais également de la topo- 
graphie. Ce maillage est construit à partir de profils terrain mesurés par la création de nœuds intermédiaires (ou nœuds de calcul), dont les caractéristiques sont interpolées à partir des profils terrain. II sera aussi fonction des caractéristiques hydrauliques des écoulements (zones de transition entre régimes notamment). La figure 3 illustre un maillage à un carrefour sur lequel ont été reportés des profils terrain ainsi que des nœuds de calcul. Ceux-ci schématisent les profils en travers des rues qui sont représentés par un gabarit rectangulaire assorti d'un coefficient de rugosité.

Les sens d'écoulement ne sont pas préjugés : ils sont déterminés par l'hydraulicité du domaine et peuvent évidemment varier tout au long du calcul.

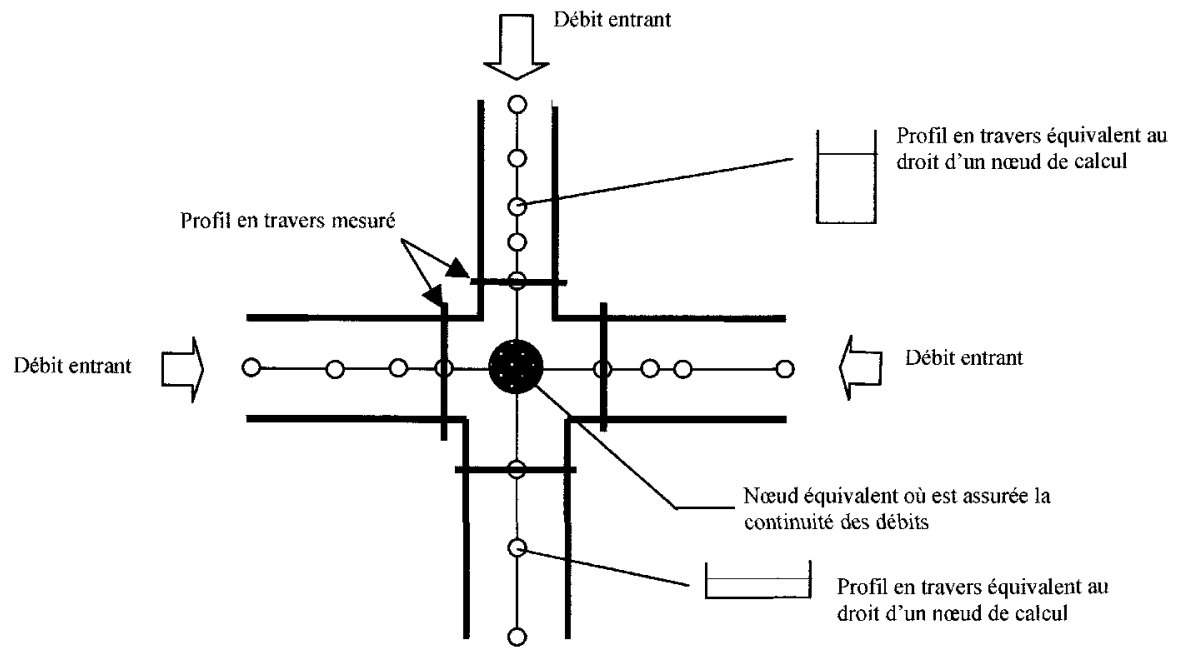

Figure 3 Schématisation d'un carrefour.

Schematisation of a crossroads.

\subsection{Mise en œuvre du modèle}

En chaque nœud du maillage et à chaque pas de temps peuvent être évalués le débit instantané ainsi que le niveau d'eau. C'est ainsi que l'on peut extraire des hydrogrammes et des limnigrammes, qui par intégration nous donnent les volumes transités par rue.

La disponibilité spatiale et temporelle des niveaux d'eau simulés permet de réaliser des images dynamiques où la propagation des eaux peut être illustrée sur un fond cartographique, qui donne un certain " réalisme " au phénomène et permet d'apprécier la dynamique des écoulements.

Dans le cadre de cette démarche exploratoire, les paramètres hydrauliques n'ont pas fait l'objet d'une analyse de sensibilité. Leurs valeurs ont été fixées à $K=40 \mathrm{~m}^{1 / 3} \mathrm{~s}^{-1}$ pour le coefficient de rugosité de Strickler et à $v_{t}=1 \mathrm{~m}^{2} / \mathrm{s}$ pour la dispersion.

Le maillage unidimensionnel est constitué de 368 profils, de 329 branches (ou biefs) et de 147 jonctions (carrefours). 
D'une manière générale, les niveaux atteints sont assez proches des mesures compte tenu de l'absence de calage des paramètres. La violence même du phénomène explique en grande partie ces bons résultats. Pour des phénomènes moins violents, les processus sont beaucoup moins tranchés et l'écoulement devient alors plus sensible aux singularités, au stockage dans les maisons, aux échanges avec les réseaux, etc.

Les meilleurs résultats sont obtenus en périphérie du modèle, à proximité de l'entrée et de la sortie. Au centre du domaine, les niveaux calculés s'écartent des mesures. II est très difficile de se prononcer sur l'origine de ces divergences, qui peuvent être de natures différentes : mauvaise représentation de la topographie, non prise en compte de certaines rues adjacentes, etc.

\section{4 - MODÉLISATION BIDIMENSIONNELLE}

\subsection{Présentation du logiciel RUBAR 20}

Le logiciel RUBAR 20 résout les équations de Saint Venant bidimensionnelles y compris lorsque les variations temporelles des caractéristiques hydrauliques sont importantes (crues éclair, ondes de rupture des barrages).

Ces équations sont écrites sous la forme:

$$
\begin{aligned}
& \frac{\partial h}{\partial t}+\frac{\partial Q_{x}}{\partial x}+\frac{\partial Q_{y}}{\partial y}=P \\
& \frac{\partial Q_{x}}{\partial t}+\frac{\partial\left(\frac{Q_{x}^{2}}{h}+g \frac{h^{2}}{2}\right)}{\partial t}+\frac{\partial\left(\frac{Q_{x} Q_{y}}{h}\right)}{\partial y}= \\
& -g h \frac{\partial Z}{\partial x}-g \frac{Q_{x} \sqrt{Q_{x}^{2}+Q_{y}^{2}}}{K_{s}^{2} h^{7 / 3}}+K\left(\frac{\partial}{\partial x}\left(h \frac{\partial\left(Q_{x} / h\right)}{\partial x}\right)+\frac{\partial}{\partial y}\left(h \frac{\partial\left(Q_{x} / h\right)}{\partial y}\right)\right)+F_{x}(V)+P_{x} \\
& \frac{\partial Q_{y}}{\partial t}+\frac{\partial\left(\frac{Q_{x} Q_{y}}{h}\right)}{\partial x}+\frac{\partial\left(\frac{Q_{y}^{2}}{h}+g \frac{h^{2}}{2}\right)}{\partial y}= \\
& -g h \frac{\partial Z}{\partial y} g \frac{Q_{y} \sqrt{Q_{x}^{2}+Q_{y}^{2}}}{K_{s}^{2} h^{7 / 3}}+K\left(\frac{\partial}{\partial x}\left(h \frac{\partial\left(Q_{y} / h\right)}{\partial x}\right)+\frac{\partial}{\partial y}\left(h \frac{\partial\left(Q_{y} / h\right)}{\partial y}\right)\right)+F_{y}(V)+P_{y}
\end{aligned}
$$

où $h$ est la hauteur d'eau, $Z$ la cote du fond, $Q_{x}$ le débit unitaire selon l'axe $O x$ égal au produit de la vitesse par la hauteur d'eau, $Q_{y}$ le débit unitaire selon Oy, $g$ l'accélération de la pesanteur, $K$ un coefficient de viscosité (ou diffusion), $K_{s}$ le coefficient de Strickler, $V$ la vitesse du vent, $F_{x}$ et $F_{y}$ des relations exprimant 
les contraintes dues au vent sur la surface de l'eau, $P_{x}$ et $P_{y}$ les composantes du frottement à la paroi (verticale) exprimées sous une formulation de type Chézy ou Strickler, $P$ l'apport local correspondant à la pluie (positif) ou à l'infiltration (négatif).

Le code de calcul utilise la méthode des volumes finis appliquée à un maillage non structuré constitué de quadrilatères et de triangles qui ont entre eux 0 ou 1 (entier) côté commun.

Deux schémas numériques sont disponibles pour résoudre le système d'équations $(11)+(12)+(13)$ :

- un schéma de type VAN LEER (1979) avec deuxième ordre en temps et en espace ;

- un schéma de type VAN LEER avec deuxième ordre en espace et premier ordre en temps dérivé du précédent qui ne sera pas détaillé ci-après.

Le premier schéma inclut quatre étapes (PAQUIER, 1995) :

- Un calcul du gradient de chacune des 3 variables $h$ (en fait remplacée par $z$ niveau d'eau), $Q_{x}$ et $Q_{y}$ dans chaque maille pour chacune des deux directions correspondant aux axes Ox et Oy par une méthode des moindres carrés. Pour garder le caractère de schéma à variation totale décroissante, une limitation des gradients est effectuée qui permet d'éviter la création d'extremums sur ces variables aux milieux des arêtes de la maille considérée.

- Un calcul de la variable $W=\left(h, Q_{x}, Q_{y}\right)$ à un temps intermédiaire $t_{n+1 / 2}$ au milieu $m_{i j}$ de chacune des arêtes de chaque maille $i$ par :

$$
W_{m_{i j} L}^{n+1 / 2}=W_{m_{i j} L}^{n}-0,5 \Delta t\left[f_{1}^{\prime}\left(W_{i}^{n}\right) W_{x i}^{n}+f_{2}^{\prime}\left(W_{i}^{n}\right) W_{y i}^{n}\right]+0,5 \Delta t S_{i}^{n}
$$

dans laquelle $f_{1}$ (respectivement. $f_{2}$ ) sont les flux selon Ox (respectivement $O y$ ) correspondant au premier membre des équations, $S$ le second membre, $W_{x i}$ (respectivement $W_{y i}^{m}$ ) le gradient de $W$ selon l'axe Ox (respectivement $O y$ ), l'in$\operatorname{dex} L$ (respectivement $R$ ) représente les valeurs à gauche (respectivement à droite) de l'arête puisque a priori deux valeurs différentes sont obtenues si le calcul est effectué dans la maille $i$ ou dans la maille $j$.

- La résolution d'un problème de Riemann unidimensionnel dans la direction normale à l'arête à $t_{n+1 / 2}$ afin d'estimer les flux à travers les arêtes pour le premier membre des équations. Ce mode de calcul permet de tenir compte de discontinuités de la ligne d'eau ; il permet, de ce fait, de représenter des ressauts hydrauliques sans toutefois tenir compte de leur longueur (PAQUIER, 1995). Ce problème est résolu par une linéarisation de ROE (1981) qui donne directement la valeur de ces flux.

- L'intégration du second membre sur la surface de la maille afin d'ajouter la contribution correspondante et d'obtenir la valeur finale $W_{i}^{n+1}$ par :

$$
W_{i}^{n+1}=W_{i}^{n}+\sum_{j} \frac{\varepsilon_{i j} I_{i j}}{A_{i}} \Delta t f_{1}\left(W_{m_{i j} L}^{n+1 / 2}\right)+S_{i}^{n+1 / 2} \Delta t
$$

dans laquelle $f_{1}$ représente le flux selon $O x$, la sommation portant sur les mailles $j$ qui ont un côté commun avec la maille $M_{i}$ (de surface $A_{i}$ ) et $\varepsilon_{i j}$ vaut 1 
ou - 1 selon l'orientation de l'arête $m_{i j}$ (de longueur $l_{i j}$ ) commune aux mailles $M_{i}$ et $M_{j}$.

La contribution du second membre inclut :

- Des termes de gravité ou de pente $\left(-g h \frac{\partial Z}{\partial x}\right.$ or $\left.-g h \frac{\partial Z}{\partial y}\right)$. Ces termes sont traités comme les flux afin qu'une surface libre horizontale le reste en l'absence d'apports. Par exemple, l'intégrale sur une maille $\iint-g h \frac{\partial Z}{\partial x} d x d y$ devient :

$$
0,5 \sum_{\text {artes }} \alpha_{i} L_{i}\left(h_{c}+h_{i}\right)\left(Z_{i}-Z_{c}\right) g
$$

dans laquelle $\alpha_{i}$ est la coordonnée selon $O x$ de la normale à l'arête $i, L_{i}$ est la longueur de l'arête, $h_{c}$ et $Z_{c}$ respectivement la hauteur d'eau et la cote du fond au centre de la maille, $h_{i}$ et $Z_{i}$ respectivement la hauteur d'eau et la cote du fond au milieu de l'arête $i$;

- Des termes de frottement au fond qui sont simplement estimés au centre de la maille. Leur calcul utilise toutefois une implicitation (PAQUIER, 1995) afin d'éviter des instabilités numériques quand un brusque changement intervient sur les variables.

- Des termes de diffusion qui sont traités comme des flux. En premier, les dérivées sont calculées au centre de maille puis ces dérivées sont traitées de manière identique aux variables de base pour calculer le gradient qui sera la dérivée seconde recherchée.

- Les autres termes sont simplement calculés au centre de maille et traités de manière explicite.

Les mailles sèches sont traitées de manière spécifique dans le sens où une maille est considérée comme restant (respectivement devenant) sèche tant que (respectivement dès que) la hauteur d'eau ne dépasse pas (respectivement plus) une valeur minimale $(0,1 \mathrm{~mm}$ en général).

La stabilité numérique est généralement obtenue en limitant à chaque pas de temps le nombre de Courant maximal à 1 . Pour une maille, le nombre de Courant $C_{t}$ est calculé par :

$$
C_{t}=\max _{i}\left(v_{i}+\sqrt{g h}\right) \frac{\Delta t}{\Delta_{i}}
$$

dans laquelle $i$ prend 3 ou 4 valeurs, $v_{i}$ est le module de la vitesse dans une direction $i$ normale à une des arêtes de la maille, $\Delta_{i}$ la dimension de la maille dans cette direction et $\Delta t$ le pas de temps.

En pratique, le pas de temps peut être soit fixe, soit variable (lié au nombre de Courant) ; des variations trop brutales engendrent cependant des perturbations numériques. II en est de même des dimensions de maille qui ne doivent pas être dans un trop grand rapport entre les deux directions et d'une maille à sa voisine ; toutefois, sur le maillage présenté ci-dessous, un rapport de l'ordre de 200 n'a pas semblé générer d'instabilités notables. 


\subsection{Mise au point du modèle topographique utilisé pour les simulations}

Afin d'effectuer une comparaison plus simple avec la modélisation unidimensionnelle, seul le réseau de rues a été modélisé, ce qui réduit, en partie, la représentativité du modèle bidimensionnel dans un milieu urbain varié et concentre sa différence par rapport au modèle unidimensionnel sur la représentation des carrefours.

Comme expliqué précédemment, à l'origine tous les profils ont été décrits avec un nombre de points compris entre 11 et 19 (généralement 13). À partir de ces profils, on a supprimé des points afin d'obtenir uniformément un profil comportant 11 points (figure 4).

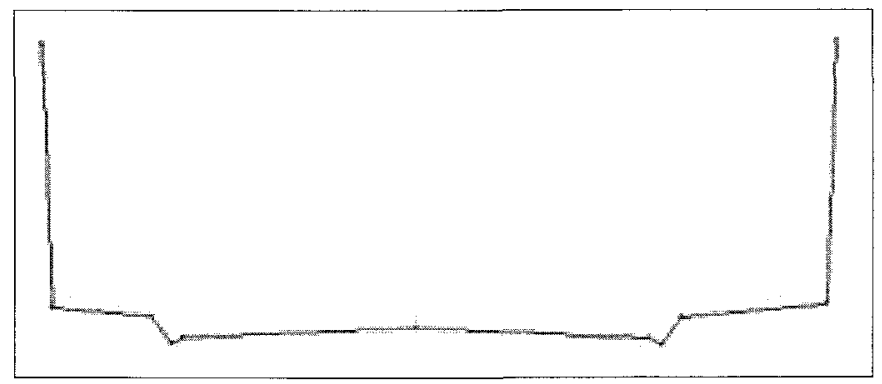

Figure 4 Profil en travers type à 11 points.

Typical cross profile with 11 points.

Des simplifications successives de ces profils ont conduit ensuite à :

- un profil à 7 points lorsqu'on supprime les deux paires de points correspondant au bas du trottoir et au début de la chaussée des deux côtés de l'axe de la rue;

- un profil à 5 points qui ne contient que les deux murs décrits avec deux points chacun plus le point de l'axe de la chaussée ;

- finalement, le profil à 4 points est dérivé de celui à 5 points en supprimant l'axe de la rue. Ce dernier profil correspond à la description minimale d'une rue. L'addition de points supplémentaires correspond à différents niveaux de détail mais ne change pas la structure générale du maillage. En examinant l'effet du nombre de points des profils en travers sur les résultats, on souhaite savoir si une description topographique détaillée amène à une plus grande précision sur les résultats.

Pour chaque type de profil en travers, un maillage a été bâti pour servir aux différentes simulations. À partir des profils en travers, une interpolation linéaire sur la cote du fond $z$ donne des profils en travers complémentaires. Par ailleurs, les données fournies ne concernaient que les rues et pour créer le maillage du calcul, on doit extrapoler ces données afin de mailler les carrefours.

Pour représenter les carrefours dans le modèle numérique, on a choisi de les interpoler à partir des profils en travers des rues dont le croisement constitue ce carrefour. 
Les profils en long d'une rue sont obtenus à partir des profils en travers de cette rue, en joignant entre eux, les points de même numéro dans chaque section. Le croisement des profils en long de deux rues sécantes donnera alors le maillage du carrefour (figure 5 par exemple). Les cotes du fond $z$ au niveau du carrefour sont ensuite corrigées pour tenir compte d'une structure idéalisée du carrefour où les bâtiments puis les trottoirs ne s'étendent que sur les angles du carrefour.

\section{rue $n^{\circ} 1$}

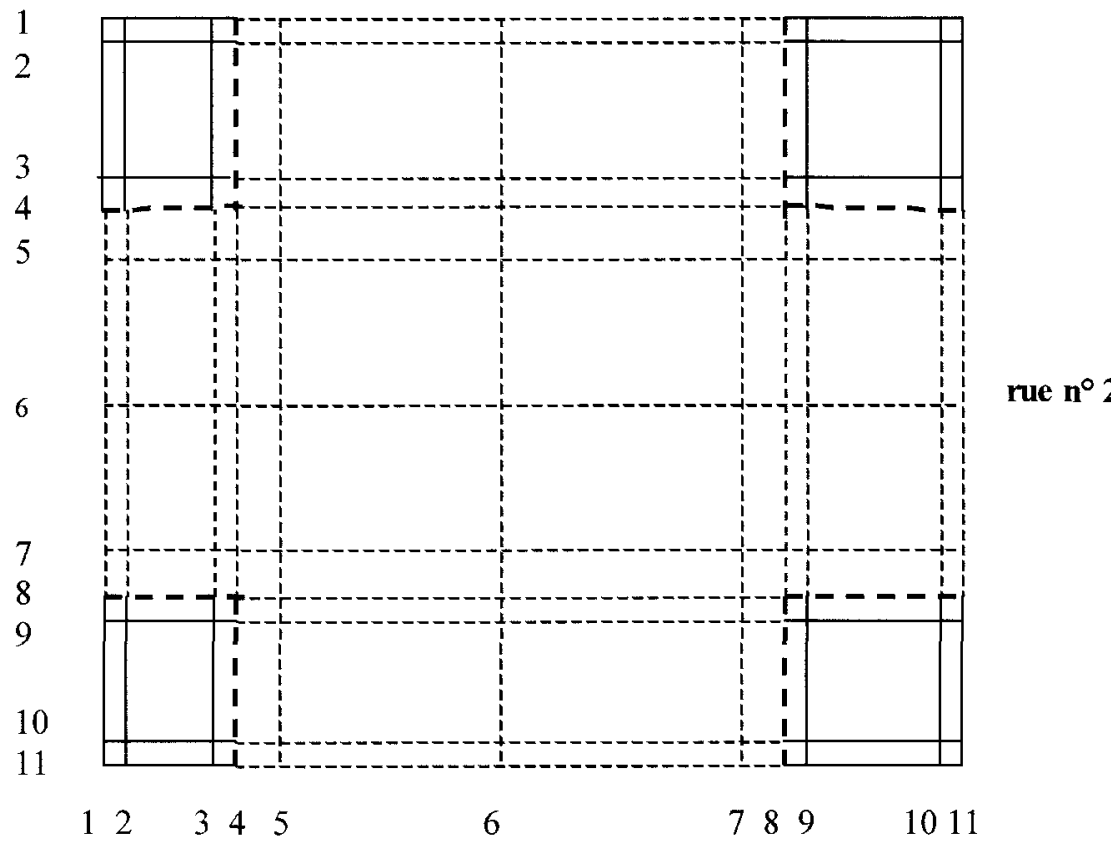

Figure 5 Carrefour de rues à 11 points.

Crossroads of 11-points streets.

Pour les carrefours plus complexes, le maillage a été construit manuellement, chaque cas apparaissant comme un cas particulier. Le maillage final se présente alors comme un quadrillage de rues, soit orientées Nord-Sud, soit orientées Est-Ouest (figure 6).

Afin d'assurer une stabilité numérique suffisante, le pas d'espace le long des rues est limité à $50 \mathrm{~m}$ avec une moyenne de l'ordre de $25 \mathrm{~m}$. Transversalement, en revanche, le pas d'espace peut être beaucoup plus faible puisqu'il est au minimum de $10 \mathrm{~cm}$. Le pas de temps est gardé fixe et égal à 0,01 seconde. Les conditions initiales des simulations sont fixées à un modèle vide correspondant à la situation avant la crue. Les conditions aux limites amont sont un hydrogramme à chaque entrée ; en outre, pour les cas où le régime devient torrentiel, la hauteur d'eau a été fixée à une valeur de l'ordre d'un mètre (mais cette valeur a peu d'influence). À l'aval, seules les rues débouchant vers le Sud ont été définies comme sorties. Ces sorties s'effectuant souvent en régime torrentiel ont été définies comme sorties libres, ce qui suppose l'absence d'in- 


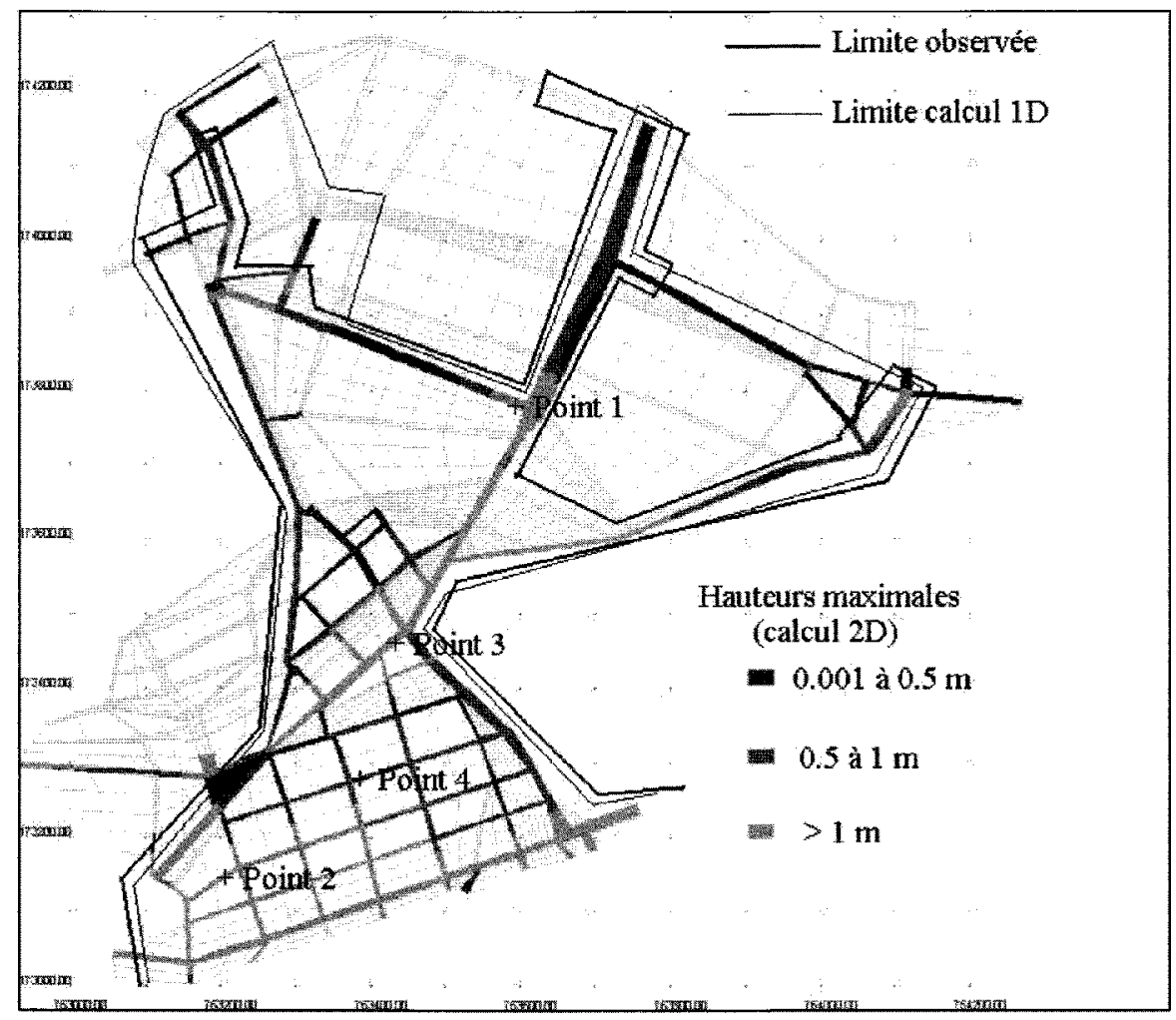

Figure 6 Maillage de calcul et hauteurs d'eau maximales du calcul 2D et limites approximatives des zones inondées observées ou estimées à partir du calcul 1D.

Calculation grid and peak water depths of the $2 D$ calculation and approximate limits of the flooded areas either observed or estimated from $1 D$ calculation.

fluence du niveau aval (en dehors du modèle) ; afin que cette hypothèse soit assez bien vérifiée, on a déplacé la limite aval vers le Nord (c'est-à-dire le long de la rue Pierre Semard) afin d'éviter l'influence du remblai ferroviaire situé au Sud.

Les coefficients de frottement au fond (Stricklers) ont été égalés uniformément à $40 \mathrm{~m}^{1 / 3} / \mathrm{s}$. Les influences de la pluie, du vent et du frottement contre le bâti ont été négligées. Le coefficient de diffusion est également pris nul dans les calculs présentés ; une analyse de sensibilité a montré que l'augmentation de ce coefficient jouait sur l'amortissement de la crue et l'élévation des niveaux, un rôle un peu différent d'une augmentation du frottement au fond ; toutefois, la dynamique générale de la crue et le niveau moyen des eaux ne sont pas suffisamment affectés pour modifier les enseignements tirés aux paragraphes 5 et 6 . 


\section{5 - RÉSULTATS DES MODÈLES ET COMPARAISON AVEC LES OBSERVATIONS}

Compte tenu des données de validation disponibles, la comparaison des résultats des simulations avec les observations ne peut avoir lieu qu'en terme d'extension de la zone inondée et du niveau d'eau maximal.

L'extension générale de la zone inondée (figure 6) est bien reproduite par les modèles pour autant qu'on puisse comparer ces diverses estimations. Ceci n'est pas étonnant car elle est très liée à la topographie du site et à la localisation des entrées en amont des deux principaux talwegs. Des différences ponctuelles existent à la fois entre modélisations numériques et observations et entre les différentes modélisations. Elles sont, en premier lieu, dues aux simplifications effectuées pour les modélisations : par exemple, absence de prise en compte de certaines rues, simplification (linéarisation) du profil en long. Les différences en limites extérieures de zones peuvent aussi être le reflet de différences de niveau du sol entre le modèle numérique et la réalité (écart type de $0,26 \mathrm{~m}$ sur la cote du fond aux points d'observation entre modèle bidimensionnel et mesures) ou entre modèles.

Les niveaux d'eau maximaux simulés peuvent être comparés aux laisses de crue. Dans les lieux de passage des forts écoulements (contrairement aux zones de stockage), la hauteur d'eau maximale constitue un meilleur point de comparaison. C'est ce qui a été choisi sur les figures 7, 8, 9 et 10.

On observe qu'au point 1 (voir localisation en figure 6) situé pratiquement dans l'axe d'écoulement Est, l'accord est acceptable pour les deux modélisations malgré le retard sensible du modèle $1 \mathrm{D}$ par rapport au modèle $2 \mathrm{D}$. On retrouve ce bon accord au point 2 situé à l'extrémité Sud-Ouest de la zone modélisée en $2 \mathrm{D}$; mais contrairement au point 1 , le modèle $1 \mathrm{D}$ y est en phase avec le modèle 2D. En revanche, aux points 3 et 4 situés plus au Nord-Est (près de la gare de marchandises), il y a de fortes différences de hauteur d'eau à la fois entre modèles et entre modèles et laisses de crue. Le modèle $2 D$, en particulier, donne des hauteurs d'eau faibles qui se maintiennent alors que le modèle 1D donne un maximum plus élevé. La comparaison ponctuelle avec les laisses de crue doit être relativisée car les laisses de crue donnent des hauteurs très variables dans l'espace $(1,5 \mathrm{~m}$ à $20 \mathrm{~m}$ du point 3 et $1,7 \mathrm{~m}$ aux carrefours voisins du point 4). Les différences constatées entre modèles semblent tenir essentiellement aux pertes de charge soit le long des rues (coefficient de Strickler pris identique mais ayant une signification légèrement différente en 1D et en 2D) soit aux carrefours où, en outre, la répartition des débits est fondamentalement différente entre les modèles puisqu'en 2D, la topographie du carrefour et l'orientation des voies jouent un rôle important alors qu'il n'en est pas tenu compte en $1 \mathrm{D}$.

Sur la base d'un relativement grand nombre de laisses de crue (83 disponibles sur le secteur), le constat est : la hauteur d'eau maximale moyenne sur toute la zone d'étude se situe, pour le calcul 2D, entre 12 et $22 \mathrm{~cm}$ (selon le nombre de points) au-dessous des hauteurs d'eau maximales déterminées à partir des laisses de crue ; l'écart quadratique moyen correspondant est de l'ordre de $80 \mathrm{~cm}$. Cet écart ne varie que faiblement $(1 \mathrm{~cm})$ lors d'une augmentation des débits de $40 \%$ ou lors du passage à un frottement plus fort (Strick- 


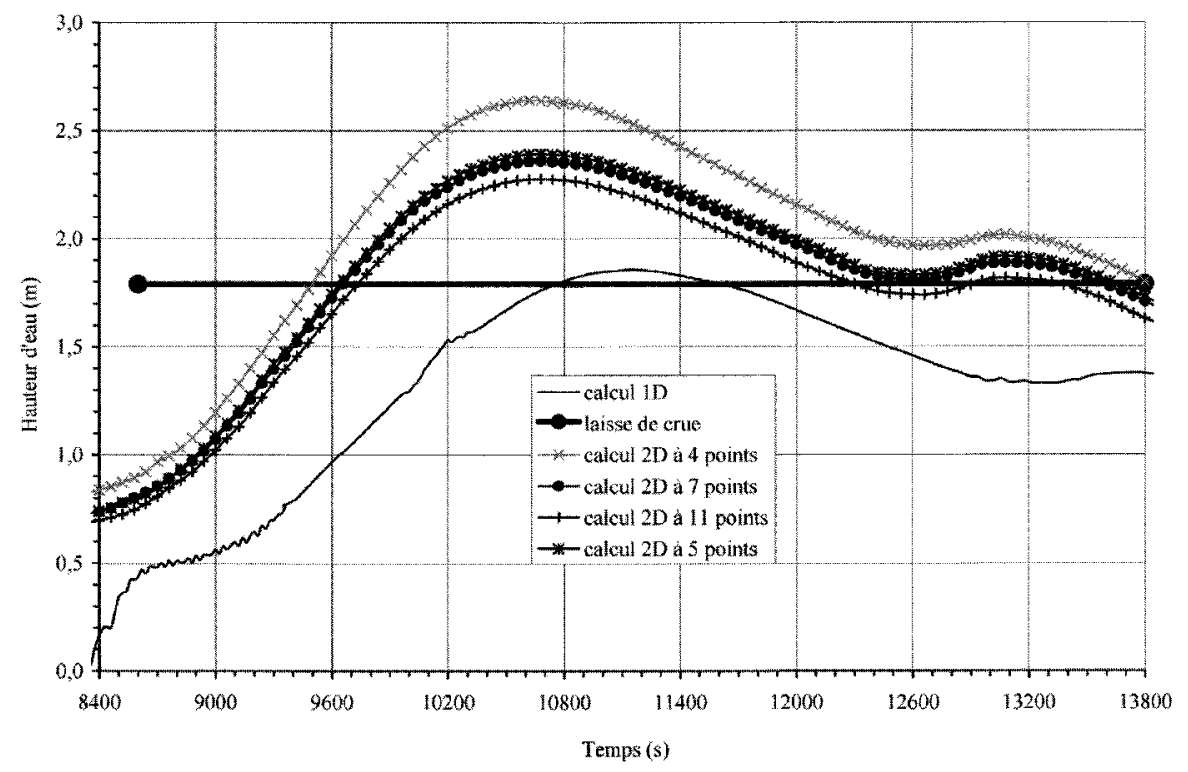

Figure $7 \quad$ Limnigrammes au point 1.

Water depths at point 1.

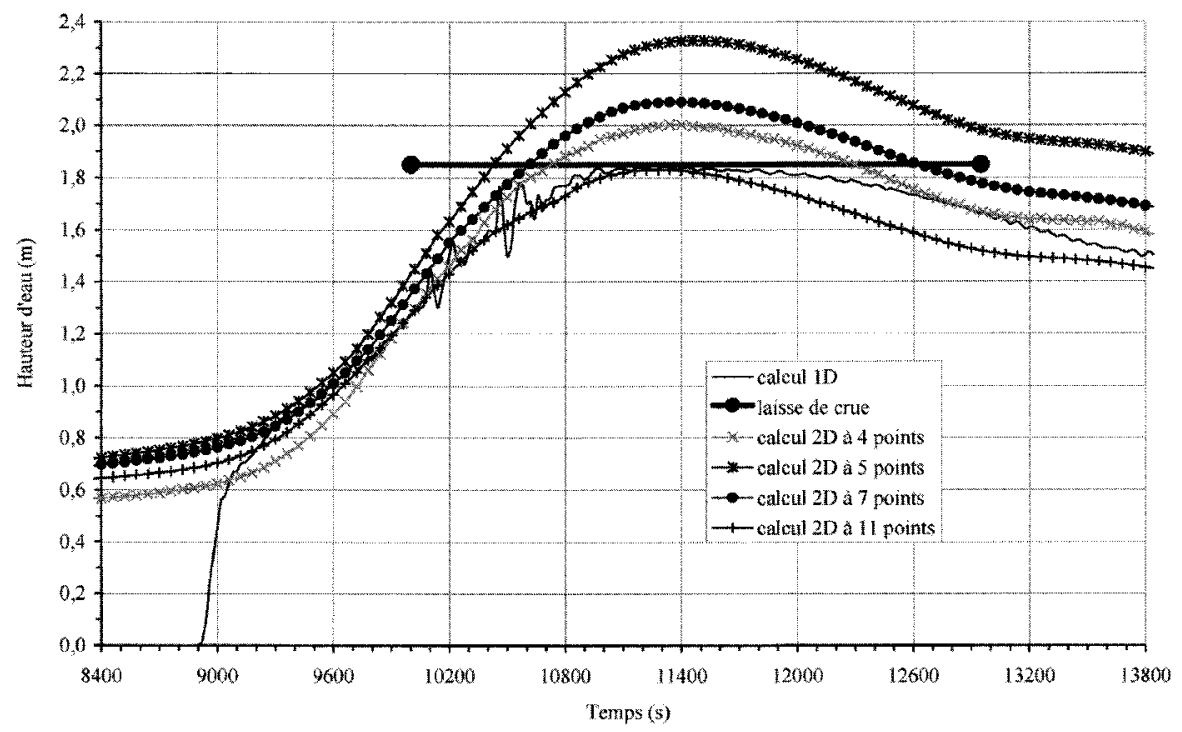

Figure $8 \quad$ Limnigrammes au point 2.

Water depths at point 2. 


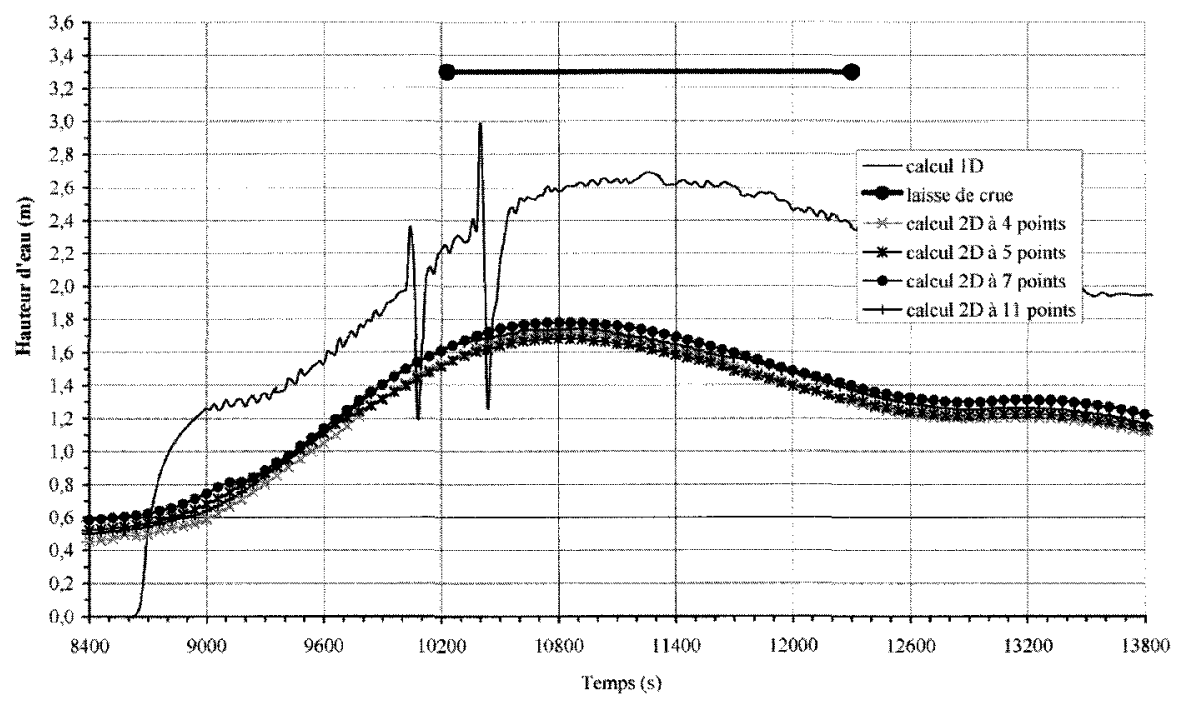

Figure $9 \quad$ Limnigrammes au point 3.

Water depths at point 3.

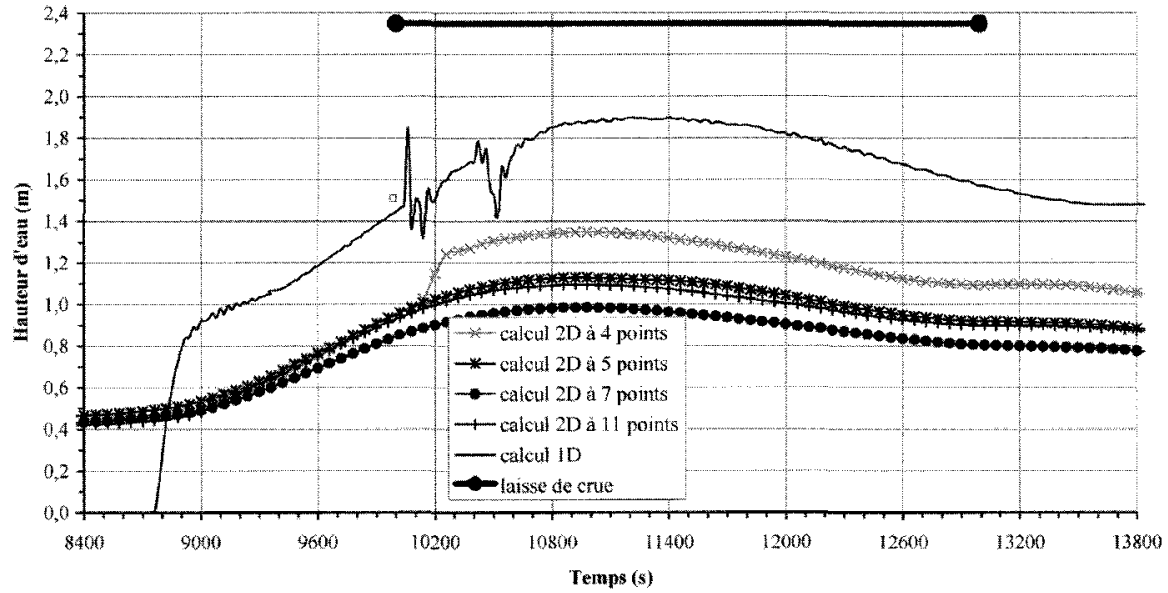

Figure 10 Limnigrammes au point 4.

Water depths at point 4.

ler de 30) ce qui peut permettre de caler la hauteur maximale moyenne calculée sur la valeur observée. Sur un échantillon de 10 points supposé représentatif et s'appuyant sur des laisses de crue supposées plus fiables (corrélation avec observations voisines en particulier), le calcul 1D donne une moyenne trop faible de $5 \mathrm{~cm}$ et un écart type de $30 \mathrm{~cm}$. Un résultat équivalent est obtenu par le calcul 2D. 
Une partie de ces écarts est due à l'imprécision des données topographiques et à la différence de localisation entre points de calcul et points d'observation ; ceci est reflété par l'écart type de l'ordre de $0,3 \mathrm{~m}$ sur les cotes du fond du modèle $2 \mathrm{D}$ (sur 83 points) et par les différences entre résultats du modèle $2 \mathrm{D}$ avec des nombres de points différents qui conduisent à une hausse du niveau moyen du fond de $6 \mathrm{~cm}$ et une hausse du niveau maximal moyen de l'eau de $16 \mathrm{~cm}$ en passant de 11 points à 4 points. Une seconde part est due aux obstacles locaux, aux vagues et autres fluctuations locales de niveau (réelles ou dues à l'imprécision des observations) ; ainsi, alors que les modèles numériques donnent un niveau similaire, deux laisses de crue de part et d'autre d'une rue peuvent différer de plus de $50 \mathrm{~cm}$ en niveau. Enfin, la troisième part, sans doute la plus importante est due aux modèles eux-mêmes, y compris les simplifications effectuées dans la description du milieu (hypothèse d'un bâti continu et imperméable en premier lieu) et des processus (absence de prise en compte de la pluie sur la zone considérée et des écoulements transités par le réseau d'assainissement) ; cela est notable au vu des résultats sensiblement différents quand on modifie le nombre de points pas section de rue dans le modèle $2 \mathrm{D}$ mais aussi, à travers différents tests de sensibilité effectués : par exemple, dans la modélisation 2D, ajouter l'élargissement d'une rue constituée par une petite place peut diminuer localement le niveau de $10 \mathrm{~cm}$, ajouter une rangée de voitures en stationnement peut augmenter dans la rue le niveau de $10 \mathrm{~cm}$ (HAIDER, 2001).

\section{6 - CONCLUSIONS}

Les dommages dus aux inondations sont, en grande partie, concentrés dans des milieux urbanisés (centre ville ou périphérie). La simulation des écoulements dans de tels milieux est nécessaire pour établir des mesures de prévention ou de prévision. La complexité de ces écoulements, du fait du grand nombre d'obstacles et de bifurcations, exige le développement de modèles numériques adaptés qui puissent, en particulier, traiter les passages entre régimes torrentiel et fluvial. Le cas de la crue de Nîmes du 3 octobre 1988 qui comporte des écoulements rapides (vitesse maximale supérieure en maints endroits à $2 \mathrm{~m} / \mathrm{s}$ ) a montré que les codes $1 \mathrm{D}$ maillé $\mathrm{REM}^{2} \mathrm{U}$ et $2 \mathrm{D}$ Rubar 20 permettent de tels calculs dans un réseau de rues moyennant un choix convenable des pas de temps et d'espace. Une analyse de sensibilité exhaustive reste toutefois à effectuer pour déterminer les paramètres optimaux, en particulier, en fonction des simplifications effectuées. Le code 2D requiert des temps de calcul importants (de l'ordre de la journée pour le calcul de 4 heures de crue). II est potentiellement capable de traiter des milieux plus hétérogènes sans hypothèse supplémentaire alors que des améliorations relatives au traitement des carrefours et des zones de stockage telles que parcs, terrains de jeux, aires de stationnement sont encore à intégrer dans le code 1D; de ce fait, la modélisation 2D qui permet de décrire directement la vue en plan apparaît plus simple d'emploi même si une sélection doit toujours être effectuée parmi les détails à représenter dans le modèle. Le code 1D reste l'outil privilégié pour représenter les écoulements dans les réseaux de rues, à l'intérieur desquelles les écoulements sont plutôt 
unidimensionnels ; la modélisation globale d'un quartier très urbanisé où les linéaires de voies sont très importants pénalise d'ailleurs le modèle 2D. Compte tenu des intérêts complémentaires des deux types de modélisation, il conviendrait de développer un code mixte 1D-2D complet, dont certains nœuds représenteraient des profils en travers et d'autres ncuds seraient des points d'un maillage surfacique. Les rues uniformes seraient alors maillées en $1 \mathrm{D}$ et les carrefours ou les points singuliers en $2 \mathrm{D}$.

Pour les deux codes, un prétraitement plus efficace est nécessaire pour intégrer les détails du milieu urbain tels qu'ils peuvent être appréhendés à partir des bases de données urbaines fort disparates entre les villes ou de prises de vue aériennes ; un traitement automatique de la topologie du tissu urbain, de la topographie disponible devrait permettre de rendre ces outils beaucoup plus souples d'utilisation. De même, des méthodologies adaptées restent à définir pour estimer les échanges avec le réseau d'assainissement (des traitements appropriés apparaissent dans KINOSHITA et al., 1996 ; HSU et al., 2002), les apports pluviaux et le stockage à l'intérieur du bâti. Les tests futurs incluant la comparaison des deux modèles devraient être menés sur des événements moins violents (vitesses plus faibles) s'accompagnant de profondeurs d'eau plus faibles. Ceci complètera alors la validation des outils développés et permettra d'identifier quels sont les éléments des méthodes à améliorer en priorité. En ce qui concerne le post traitement des résultats, des essais de visualisation 3D capables de représenter la dynamique de la crue sont en cours d'étude.

\section{REMERCIEMENTS}

Ce travail a été accompli dans le cadre des projets « Risque hydrologique en milieu urbain " et "Estimation des écoulements de surface pour une crue extrême en milieu urbanisé " du programme RIO (Risques d'inondation) soutenu par le ministère de l'Aménagement du Territoire et de l'Environnement et du projet « Modélisation de processus hydrauliques à surface libre en présence de singularités " de l'Action concertée incitative "Prévention des catastrophes naturelles » soutenu par le ministère de la Recherche. Nous remercions aussi tous les partenaires de ces projets.

\section{RÉFÉRENCES BIBLIOGRAPHIQUES}

AL MIKDAD O., 2000. Modélisation des écoulements en réseaux maillés et ramifiés. Thèse de doctorat, université de technologie de Compiègne, $250 \mathrm{p}$.

CAMPANA N.A., TUCCI C.E.M., 2001. Predicting floods from urban development scenarios: case study of the Dilluvio basin, Porto Alegre, Brazil. Urban Water, 3, 113-124.

DESBORDES M., DUREPAIRE P., GILLY J.C., MASSON J.M., MAURIN Y., 1989. 3 octobre 1988: inondations sur Nîmes 
et sa région - Manifestations, causes et conséquences, Nîmes, France, Lacour, $96 \mathrm{p}$.

GALLATI M., BRASCHI G., 1990. Simulation of the inondation of large areas of complex topography caused by heavy floods. In: BLAIN E., OUAZAR D. (éd.), HYDROSOFT 90. Int. Conf. Hydraulic Engineering Software Applications, Lowell, Massachussets, États-Unis, Computational Mechanics Publications, 117-126.

HAIDER S., 2001. Contribution à la modélisation d'une inondation en zone urbanisée. Approche bidimensionnelle par les équations de Saint Venant. Thèse de doctorat, Lyon, Institut des sciences appliquées, $173 \mathrm{p}$.

HERVOUET J.-M., SAMIE R., MOREAU B., 2000. Modelling urban areas in dam-break flood wave numerical simulations. RESC$D A M$ workshop, Seinäjoki, Finland, $12 \mathrm{p}$.

HINGRAY B., BOUVIER C., CAPPELAERE B., DESBORDES M., 2000. Inondations urbaines dans les PED : un indicateur géométrique caractéristique du comportement hydrodynamique du bâti. Revue des Sciences de l'Eau, 13 (1), 85-100.

HSU M.-H., CHEN S.-H., CHANG T.-J. 2002. Dynamic inundation simulation of storm water interaction between sewer system and overland flows. Journal of the Chinese Institute of Engineers, 25 (2), 171-1777.

KHAN A., CADAVID R., WANG S.S.Y., 2000. Simulation of channel confluence and bifurcation using the CCHE2D model. Water and Maritime Engineering, 142, 97-102.

KINOSHITA S., SATO S., TERAYAMA H., 1996. Flood simulation by two-dimensional tank model. 7th International Conference on Urban Storm Drainage, Hanover, Germany, 959-964.
LAI J.-S., HUANG L.-H., HSIEH P.-C., LIN M. $-Y, 2000$. Integration of models for simulating flood inundation in the urban area. Hydroinformatics 2000, lowa City, lowa, 8 p.

PAQUIER A., 1995. Modélisation et simulation de la propagation de l'onde de rupture de barrage. Thèse de doctorat, université Jean Monnet de Saint-Étienne, $215 \mathrm{p}$.

PAQUIER A., 1998. 1-D and 2-D models for simulating dam-break waves and natural floods. Concerted action on dam-break modelling, proceedings of the CADAM meeting, Wallingford, United Kingdom. In: MORRIS M., GALLAND J.-C., BALABANIS P. (éd.), European Commission, Science Research Development, Hydrological and hydrogeological risks, L2985, Luxembourg, 127-140.

RICCARDI G.A., 1997, The mathematical modelling of flood propagation for the delineation of flood risk zones. Sustainability of Water Resources under Increasing Uncertainty (Rabat Symposium S1), Rabat, Maroc, 355-363.

ROE P.L., 1981. Approximate Riemann solvers, parameter vectors and difference schemes. Journal of Computational Physics, 43, 357-372.

SMITH M., 1992. Street flow analysis using a GIS-based distributed parameter hydrologic model, Hydrocomp92, Budapest, Hungary, 391-399.

TANGUY J.M., AL MIKDAD O., ZHANG B., 2001. Risque hydrologique pluvial urbain - un outil de simulation des écoulements superficieis, Bulletin des Laboratoires des Ponts et Chaussées, 232, 85-97.

VAN LEER B., 1979, Towards the ultimate conservative difference scheme. V.A second-order sequel to GODUNOV's method. Journal of Computational Physics, 32, 101-136. 